\title{
A COMPARATIVE STUDY ON THE VISIBILITY RELATION OF VIETNAM TRADITIONAL AND CONTEMPORARY TUBE HOUSE PLANS
}

\author{
NGUYEN PHUONG THAO \& BART JULIEN DEWANCKER \\ Department of Architecture, University of Kitakyushu, Japan
}

\begin{abstract}
Vietnam is a low-income country in the world (125/185): from 1986, the pace of urbanization in Vietnam rose sharply, which caused many urban problems. Up to now, there is about 700 urban areas in Vietnam. Due to limited land in urban areas, mostly in Vietnam's current urban areas, the main architectural form is a tube house. Tube houses have become the most popular house typology in urban areas in Vietnam. There is a variety of tube house typology with different plans for usage. However, until now there has been no official research on the tube house in Vietnam. This study will analyze the usage of tube houses in Vietnam from 1986 to 2015. The aim of this research is to analyze whether there are some differences of tube house typology between 1986-2005 and 2006-2015, and to understand better the advantages and disadvantages of the tube house, as well as calculating the percentage of green space in the tube house plan. It will also show which tube house typology is better for users with limited living space. This research includes three phases: the first phase's main task is collecting different contemporary tube house plans in Hanoi in both periods; the second phase compares all the cases about functions, structure, plan and green space inside the Tube houses; the third phase finds out the basic types of tube house and makes the conclusion about the green space ratio in total for all the cases. Finally, we used Depthmap software to analyze the visibility relations by Isovist map in the tube house plan of each house, and makes the conclusion of which type has the better visual field and better use of natural light.

Keywords: urbanization, Vietnam's tube house, visibility relation, Depthmap.
\end{abstract}

\section{INTRODUCTION}

\subsection{Research background}

Urbanisation is a crucial principle of urban development; the urbanisation rate is considered as an indicator of the development capability of a nation, region or local area. In developing countries, because of the demand for making a living, people tend to leave rural areas to move to large urban areas; Vietnam is not an exception. Vietnam is a low-income country in the world (125/185) [1]; from 1986 onwards, the pace of urbanisation in Vietnam has risen sharply, which has caused many urban problems. To date, there are about 700 urban areas in Vietnam [2]. Due to limited land in urban areas, the main architectural form of urban housing is the tube house. Tube houses have become the most popular housing typology in urban areas in Vietnam. Depending on the area of land, there is a variety of tube house typologies with different plan uses. This study will analyse the plan of the tube houses in Vietnam from 1986 to 2015.

\subsection{Research location}

Hanoi is the capital of Vietnam, which is also one of the biggest cities in the country, with a long history. It was founded in 1010. Hanoi's area measures $3328.9 \mathrm{~km}^{2}$ with a total population of 7,587,800 people in 2015 [3]. There are two main reasons for choosing Hanoi as the research site. Firstly, different from other big cities such as Ho Chi Minh or Hai Phong, 
Hanoi has a variety of different development periods such as the feudal time and the French colonial period. Therefore, it has different, unique urban areas, which leads to the appearance of a variety of tube house typologies. Secondly, Hanoi is the second biggest city in Vietnam which has a high speed of urbanisation and economic development. The construction of numerous new residential areas also encourages the development of new tube house construction styles. In short, Hanoi is a suitable site for tube house research.

\subsection{Research purpose}

The aim of this research is to analyse whether there are some differences between traditional and contemporary tube houses (in the 1986-2005 and 2006-2015 periods) and understand better the advantages and disadvantages of the tube house. At the same time, it will demonstrate which tube house typology is better for users. In order to realize the aim of the research, specific objectives should be achieved: collect the tube house plans in both periods (1986-2005 and 2006-2015) in Hanoi; classify and compare the characteristics of the main typologies of the tube house in both periods; analyse the Isovist map of each typology of the tube house in both periods; compare the analysis of both periods and reach a conclusion about which period has the better plan for each typology.

\subsection{Research methodology}

This research includes three phases. The first phase's main task is to collect different contemporary tube house plans. In the second phase, we compare all the cases regards functions, structure, plan and green space inside the houses. After that, we find out what the basic type of tube housing is like and make a conclusion about the green space ratio in general. In the final phase, Depthmap software is used to analyse the visibility relations in the tube house plan of each house and make a conclusion about which type has a better visual field.

\section{TUBE HOUSES IN VIETNAM}

\subsection{Urbanization in Vietnam}

Vietnam is a developing country which has just got rid of its low-income country status and has stepped into the group of medium-income countries. Because Vietnam was an agricultural country, urbanisation in Vietnam speeds up very slowly. It was only when Vietnam's government changed the economic policy that the national market opened up to trading with other countries, and urbanisation in Vietnam started to develop fast. In 1986, Vietnam's government opened the market to other countries, which drastically changed the whole nation's economic situation. Since 1986, urban growth in Vietnam has accelerated. According to World Bank data, an average urbanisation rate of Vietnam in the period of $1999-2009$ is $3.4 \%$. Until 2013, the national urbanisation rate is $3.34 \%$. Over nearly 30 years of economic policy renovation, Vietnam's urban area systems have developed unstoppably. In 1999, Vietnam had 629 urban areas; in 2010, the number of urban areas rose up to 755. Until November 2013, Vietnam had 770 urban areas in total, including two special urban areas, 14 type- 1 urban areas, 11 type- 2 urban areas and 52 type-3 urban areas [4, pp. 32-76].

Vietnam is a developing country which has a massive population $(93,421,835$ people in 2014) and high population density of 281.55 people per $\mathrm{km}^{2}\left(47^{\text {th }}\right.$ in the world density population). Along with the speedy develop of urbanization, Vietnam also has the high urbanization speed (3.03\% in the period 2010-2015) and urban population reached $33.6 \%$ in 2015 [4, pp. 91-102]. 
Hanoi is the capital of Vietnam, with the third highest urbanization rate. Hanoi has 11 wards in total, one town and 17 districts. According to the Master Plan of Hanoi Project 2013, Hanoi's population would reach 9.1 million people by 2030. Hanoi's urban area can be divided into the following five areas: the Old quarter; the urban ring 1, 2 and 3; and district areas. In the last 10 years, the number of immigrants to Hanoi has increased dramatically; in the period of 2000-2010, the average rate of urban population growth has risen to $3.35 \%$, which demands a large number of houses [5].

\subsection{Tube houses in Hanoi}

From 1986 to the present day, tube houses have become the most popular housing typology in Hanoi city, especially in 12 wards of Hanoi. In all of the wards that are in the inner city of Hanoi, such as Hoan Kiem, Hai Ba Trung, Ba Dinh, Cau Giay and Dong Da, tube houses comprise more than $60 \%$ of housing, compared to other types of housing, such as apartments, group houses or mansions [6].

\section{CASE STUDY}

3.1 Traditional tube houses and semi-traditional tube houses in Hanoi

According to the latest survey by JICA Research Institute, Japan, group for the HAIDEP

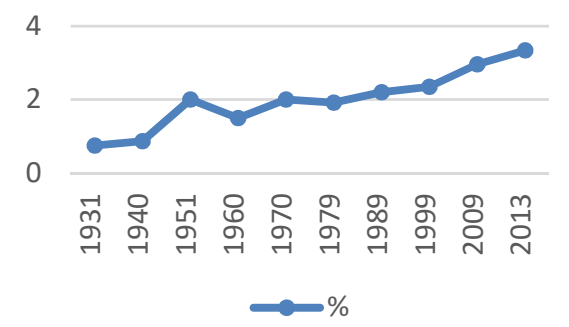

Figure 1: Urbanization rate in Vietnam from 1931 to 2013.

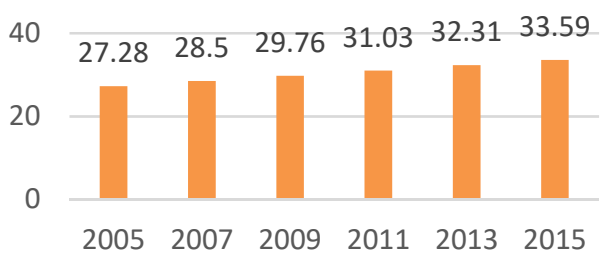

Figure 3: Vietnam population rate from 2005 to 2015 .

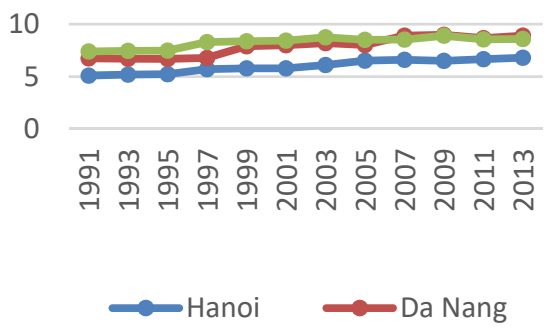

Figure 2: Urbanization rate in Hanoi, Da Nang and Ho Chi Minh city from 1991 to 2013.

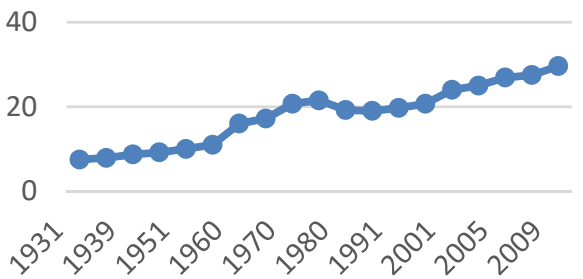

Figure 4: The percentage of urban population during 1931-2009. 
program for Hanoi, there is no longer any tube house that has the traditional structure [7]; the majority have been transformed or renovated - some of them were totally renovated, some could keep only the outer house and inside spaces were built higher, up to 3, 5 or even 7 floors.

\subsubsection{Traditional tube houses}

Fig. 5 shows a typical traditional tube house plan and a section of a house on 47 Hang Bac Street before it was renovated [8]. This is one of the oldest houses in the Old Quarter of Hanoi, and was built approximately 166 years ago according to the current owner. The house also has typical tube house architecture. The front room was generally reserved for a shop, and behind it was an open yard letting sunlight into the house. Containers to catch rainwater for washing and cooking were placed in the yard. Behind that is a storeroom, followed by a place to produce goods. Beyond that, another open yard with a kitchen and toilet. Most of the house comprised only one floor, with a roof made from red tiles sitting on high walls. Some have a small $2^{\text {nd }}$ floor with a low ceiling and only small windows, as in the Feudalism Era, but people thought it was a bad idea to look at the face of the Emperor from that height.

Traditional tube house architecture can be divided into three or four groups, and two or three inner yards placed in between, as open spaces for ventilation and natural lighting.

Studying this form signals a sustainable housing model with a system of positive characteristics. Firstly, traditional tube houses have a great eco-sufficient spatial composition that perfectly support natural ventilation and lighting.

Secondly, traditional tube houses have smart expandable spatial compositions. Fig. 6 shows two possible ways to expand the spaces (inner sky yard); i.e. when the house was becoming increasingly populated (rising number of family members, new occupants) or when new demands arose.

Option 1: Expand the land plot inward. Option 2: Build up the void spaces partially.

For option 2, it is strongly encouraged to expand only partially the voids, not entirely, in order to keep good natural ventilation and lighting. Moreover, if the voids are to be built up partly, it is highly suggested to be built on the same side, so that straight circulation and cross natural ventilation can be maintained.

Thirdly, traditional tube houses have eco-sufficient group house composition. One of particular features of Hanoi traditional tube houses is that, even when next door's house masses were not of the same sizes or position (Fig. 8) the entire system could still keep good natural ventilation within the houses and among houses, creating a self-regulated microsystem climate and preventing the heat from entering the house during summer. This is owing to the synchronized structures of the houses (alternately mass/void/mass...).

\subsubsection{Semi-traditional tube houses}

Fig. 9 shows a typical semi-traditional tube house type in the Old Quarter of Hanoi. All of the traditional houses still keep a part of the original structures and are being renovated into other architectural types in order to meet user demands. The space of the inner yards has been turned into living space. In this type, there is a very limited amount of natural light in the first and second floor of the house. Natural ventilation also is limited.

Compared to the traditional tube house, the semi-traditional house can adapt to the needs of the owners and has larger spaces for more people to live in. However, the natural lighting and ventilation quality are low. 

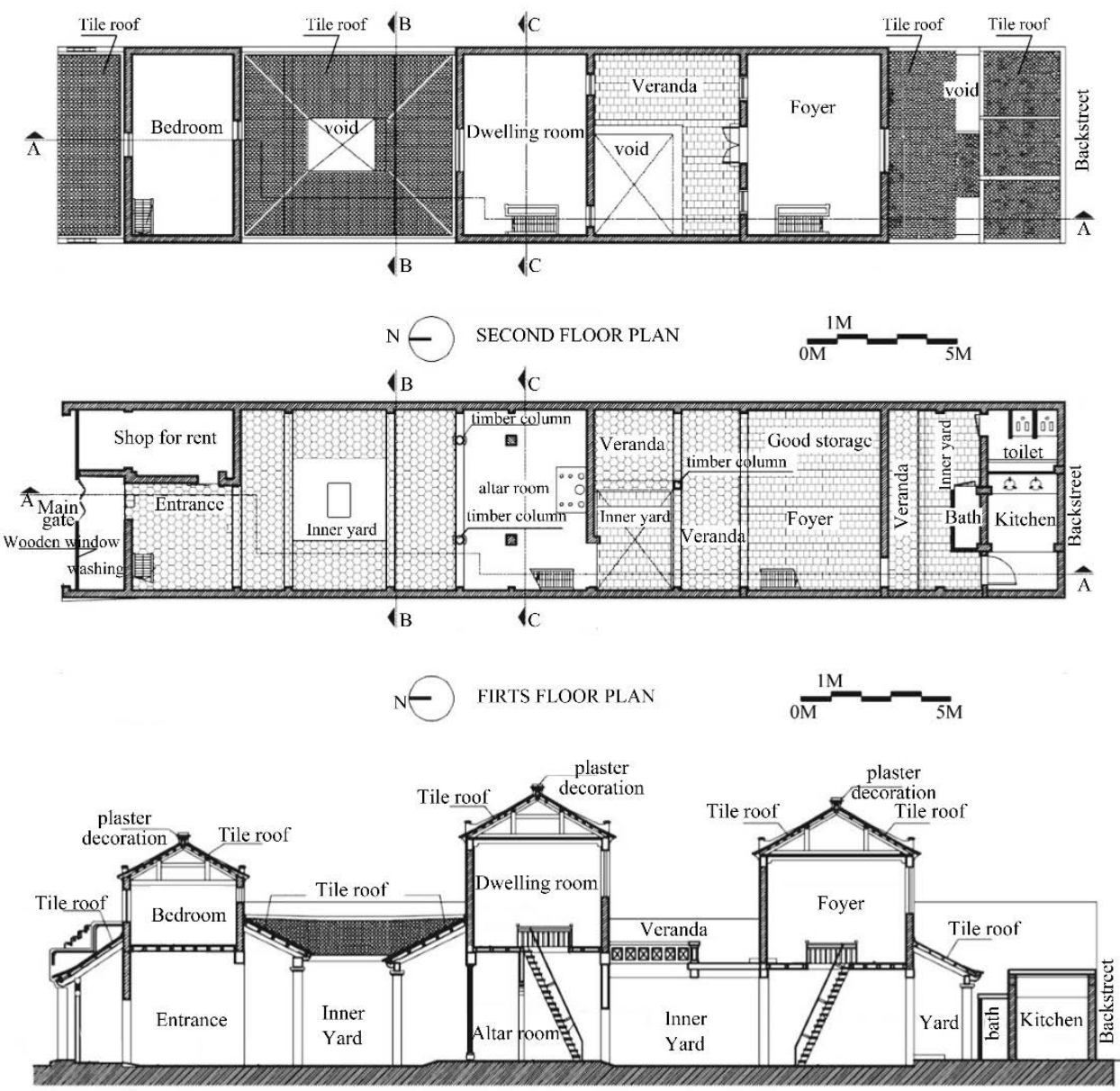

SECTION A-A

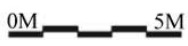

Figure 5: Traditional tube house plans and section.

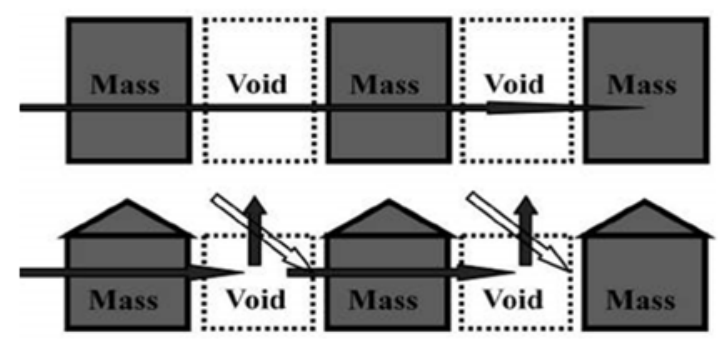

Figure 6: Tube house's eco-sufficient spatial composition. Top: Plan; Bottom: Master section; Mass: Built blocks; Void: Inner yards; Dark arrow: Natural wind; Light arrow: Natural light. 


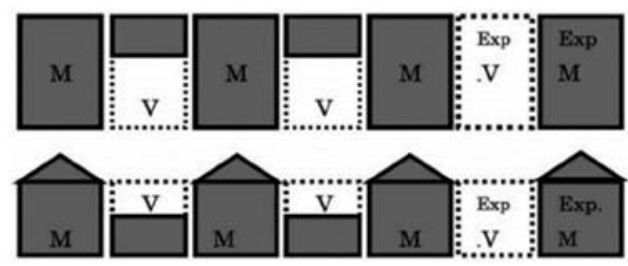

Figure 7: Tube house smart expandable spatial composition. Top: Plan; Bottom: Master section; M: Built blocks; V: Yards; Exp.: Expanded.
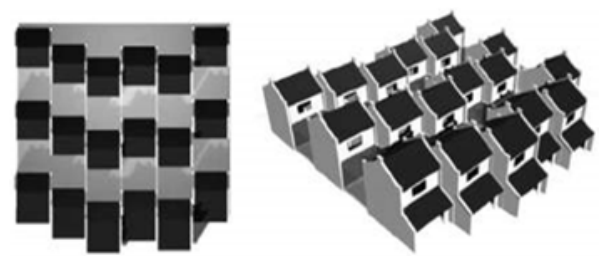

Figure 8: Diagram of a tube house group showing mass/void composition and spatial connection of open space.
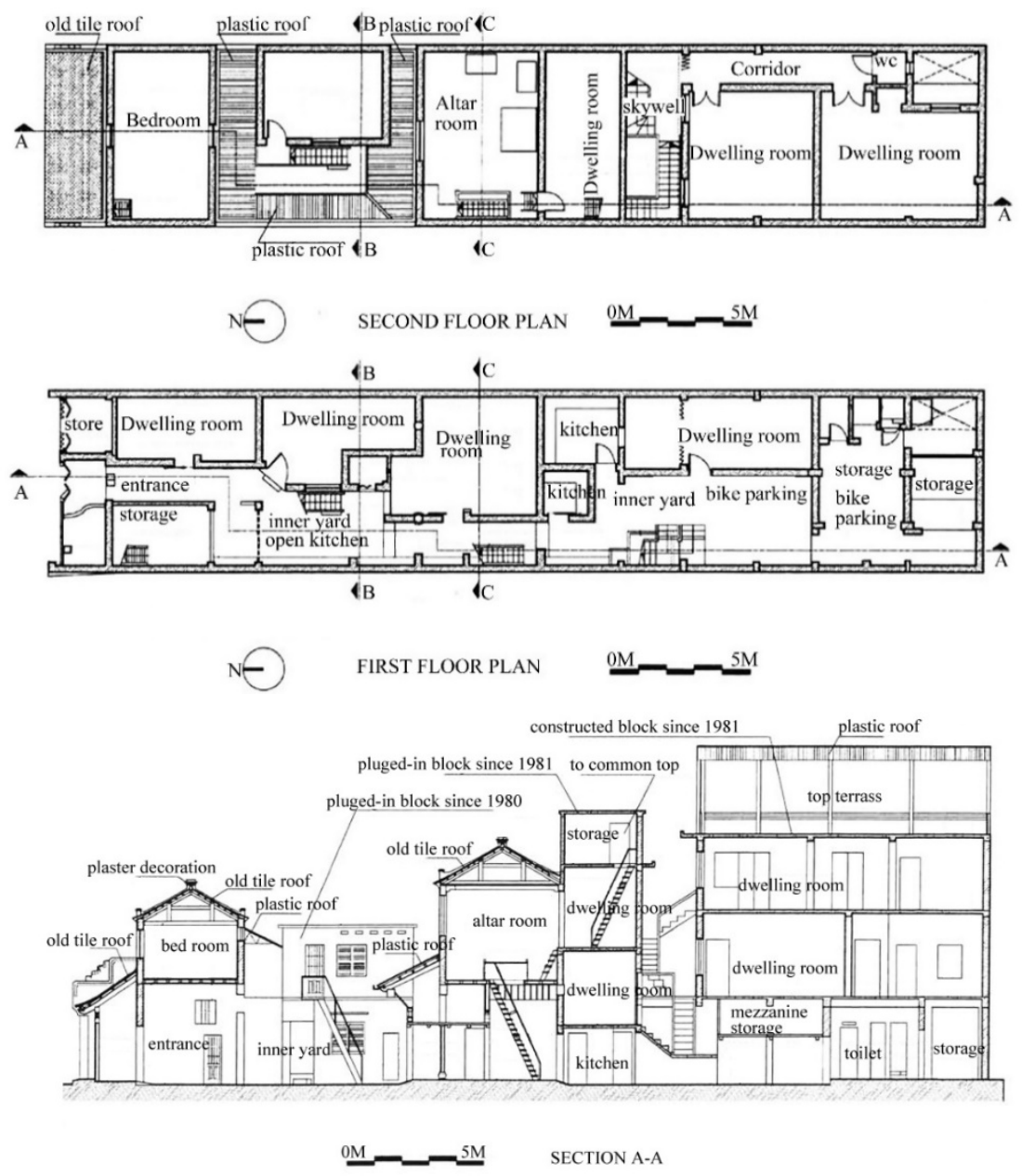

Figure 9: Semi-traditional tube house plans and sections. 


\subsection{Contemporary tube houses in Hanoi in the periods of 1986-2005 and 2006-2015}

\subsubsection{Contemporary tube house typology}

Contemporary tube houses are often called "Nhà phố" in Vietnamese and have been popular in Vietnam since 1986 within the big cities. This kind of tube house has a tube form layout which means the length or the height is much longer than the width. Contemporary tube houses are being built in all parts of the cities or urban areas in Vietnam, from large street to small alleys. If the house faces a big street, most of the front space comprises shops or offices for rent. There are very few houses that face a large street which are just residence housing.

Generally, contemporary tube houses share some basic characteristics, shaping a tube form layout; having one or more inner yards for natural lighting and ventilation; having at least one side access to the street; above land plot has narrow widths [9].

There are two ways of classifying contemporary tube houses: by morphology and by the number of open sides.

Firstly, by morphology, there are four types of contemporary tube houses: (1) full-plot built, (2) partial-plot built, (3) full-height built, and (4) partial-height built. These four types can be mixed to create many hybrid types. For example, Fig. 11 shows six hybrid types developed from the above-mentioned four types.

Originating from the traditional tube houses, and because of commercial demands, houses are now being built upward and inner yards are being minimized, even completely filled up. The houses have become multi-storey, and inner yards have become sky wells with very limited natural lighting and ventilation. The heights of the houses can be either controlled by construction regulation, or sometimes uncontrolled. Secondly, when we classify the contemporary tube house by the number of open sides, there are three basic types as shown in Fig. 12. Type 1 is the most popular. Fig. 13 shows layout examples of contemporary tube house groups. These street-front groups consist of houses of different types, different module sizes and there is almost no common rule in the designs, because most of the houses are built following the owners' needs. Compared to the traditional tube houses, the contemporary tube house group has a unique form of façades which, in turn, create a chaotic townscape. Removal of the inner yards also drives the whole neighbourhood into an entire non-selfregulated non-eco-sufficient system, which is different from the traditional neighbourhood group.

\subsection{Hanoi contemporary tube house floor plan in the periods of 1986-2005 and 2006-2015}

This research took 200 different tube houses in two periods with different layout plans and total areas. There are many functional spaces in a tube house, based on the necessity of
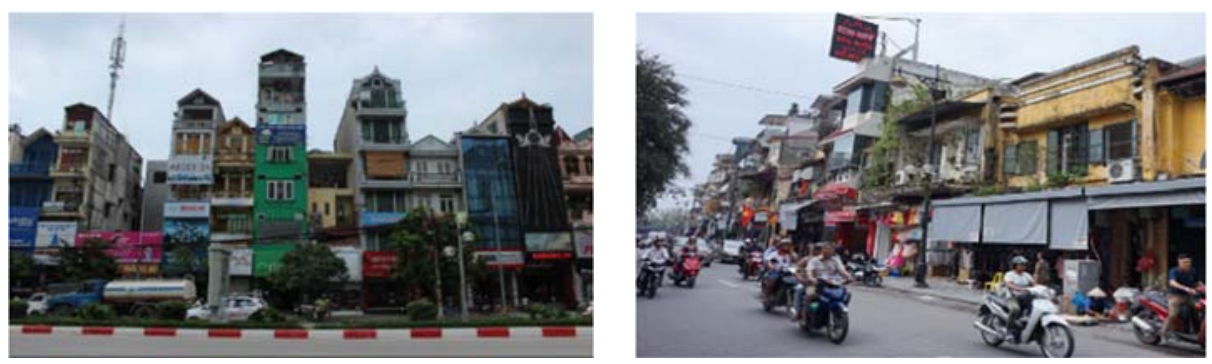

Figure 10: View of contemporary tube houses on Hanoi streets. 


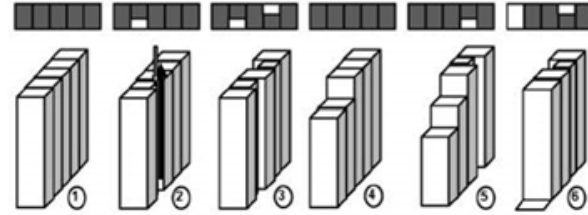

Figure 11: Contemporary tube house classified by morphology. 1 . Full plot/full height; 2. One sky well/full height; 3. Two sky wells/full height; 4. Full plot/single-step façade; 5 . One sky well/ multiple step façade; 6. One front yard/one sky well/full height.

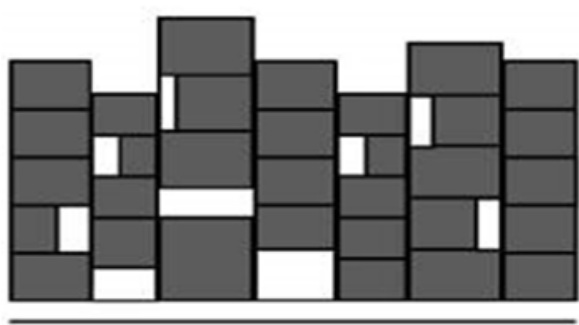

Figure 13: Example of a typical tube house group layout and a new open street crossing a tube house group in different orientation.

different functions in the layout of a tube house which is formed by the total area of each land plot; we can classify the functional space of the house into four groups. Group 1 includes all of the most basic space that a Vietnam tube house needs as common rooms (a common room can be a living room, dining room, karaoke room, even a room for parking in a limited land plot, among other functions), kitchen, bedroom, toilet, stairs, rooftop garden (which can used as a garden, laundry space, coffee yard) and space for altars. Group 2 includes the spaces that are less necessary than those in group 1 . These functional spaces include a living room, dining room, balcony, laundry room and corridor. Group 3 includes the spaces that help to improve the natural lighting of the tube house, such as an inner yard, garden, sky well and mezzanine. Group 4 includes other functions, such as working room, business, garage and storage.

Fig. 14 shows the space rates in the layout plans of contemporary tube houses in the periods 1986-2005 and 2006-2015. In the group 1, the tube houses in the $2^{\text {nd }}$ period have higher rates of common rooms and balconies; using common rooms in limited space demonstrates a better use of land efficiency, while the balconies could be used for green space and laundry. In group 2, the tube houses in the $2^{\text {nd }}$ period also have higher rates in all 
space types. The use of inner yards, skylights, mezzanines, and gardens, which enhance the house's natural light and ventilation, as well as green design appliance.

3.4 Similarities and differences between traditional and contemporary tube house characteristics

Table 1 shows the similarities and differences between two kinds of tube houses.

\section{SOFTWARE ANALYSIS}

\subsection{Isovist analysis}

Fig. 5 shows the layout plans and sections of traditional tube houses. Fig. 9 shows the layout plans and sections of semi-traditional tube house. Fig. 15 shows the layout plans and sections of a basic contemporary tube house. Compared to traditional and semi-traditional tube houses, contemporary tube houses rarely have the inner yard, but if the land plot is not too limited, most contemporary tube houses have a skylight, mezzanine, and transparent door to improve natural lighting and ventilation. Some other houses have a green wall or a doubleskin system.

After using Depthmap software to analyse the visible relationship in the layout plan and the three basic types of tube houses, the visible relationship is shown by the connectivity in Table 2.

\subsection{Results}

Fig. 16 shows the minimum connectivity in the visible relationship between the three basic types of tube houses (traditional tube house, semi-traditional tube house and contemporary tube house). The layout plans are shown in Figs 5, 9 and 15.

As we can see, traditional tube houses and semi-traditional tube houses have a very low connection with the two modern tube houses.

$(\%)$

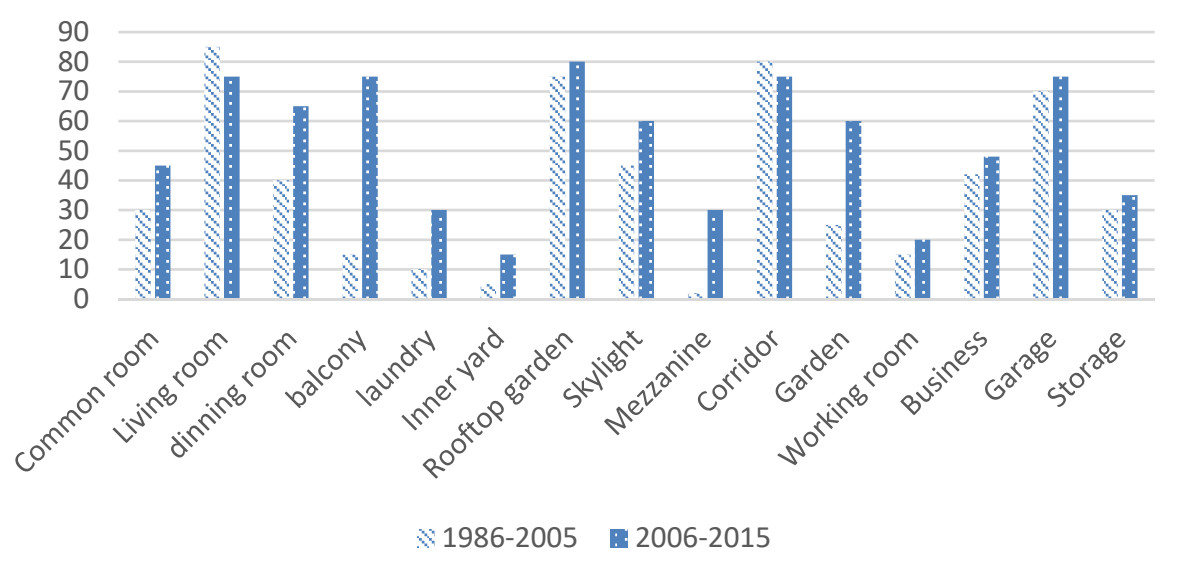

Figure 14: Space rates in the layout plans of contemporary tube houses in the periods 1986-2005 and 2006-2015. 
Table 1: Traditional and contemporary tube houses characteristics.

\begin{tabular}{|c|c|c|c|}
\hline & Type & Traditional tube house & Contemporary tube house \\
\hline 1 & Construction period & $16-19$ centuries & From late $1980 \mathrm{~s}$ \\
\hline 2 & Settlement pattern & Attached & Attached \\
\hline 3 & Average plot size & $3.5 \mathrm{~m} \times 35 \mathrm{~m}$ & $4.5 \mathrm{~m} \times 20 \mathrm{~m}$ \\
\hline 4 & Tube-form layout & Yes & Yes \\
\hline 5 & Tube-form façade & No & Yes \\
\hline 6 & Street access & Yes & Yes \\
\hline 7 & Front shop & Mostly yes & Yes/no \\
\hline 8 & Front yard & No & Mostly no \\
\hline 9 & Inner yard & Yes & Yes/no \\
\hline 10 & Number of stories & $1-2$ & $3-5$ \\
\hline 11 & Spatial structure & $\begin{array}{l}\text { Multiple alternately built } \\
\text { group yards }\end{array}$ & Mostly single-mass built group \\
\hline 12 & Building materials & $\begin{array}{l}\text { Ceramic roof tiles, } \\
\text { wooden beams, brick } \\
\text { walls, plaster }\end{array}$ & $\begin{array}{l}\text { Rein concrete bearing frame, } \\
\text { brick walls, plaster }\end{array}$ \\
\hline 13 & Technique & Manual, multi-phase & Manual, mono-phase \\
\hline 14 & Functions & Commercial + dwelling & Commercial + dwelling \\
\hline 15 & Social connection & In-house and street & Street (limited) \\
\hline 16 & Life style & Community oriented & Fairly isolated \\
\hline 17 & $\begin{array}{l}\text { Good eco-sufficient } \\
\text { spatial composition }\end{array}$ & Yes & Limited \\
\hline 18 & $\begin{array}{l}\text { Smart expandable } \\
\text { spatial composition }\end{array}$ & Yes & Yes/no \\
\hline 19 & $\begin{array}{l}\text { Eco-sufficient } \\
\text { group house } \\
\text { composition }\end{array}$ & Yes & No \\
\hline 20 & $\begin{array}{l}\text { Micro-universal } \\
\text { enjoyable inner } \\
\text { yard }\end{array}$ & Yes & Limited \\
\hline 21 & $\begin{array}{l}\text { Street as } \\
\text { community } \\
\text { connection space }\end{array}$ & Yes & Yes/no \\
\hline 22 & Human scale façade & Yes & Yes/no \\
\hline
\end{tabular}

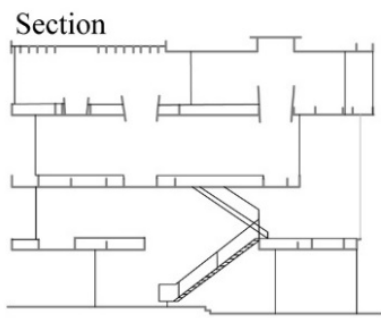

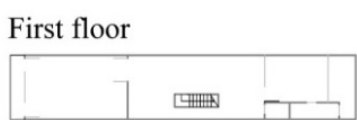

Second floor

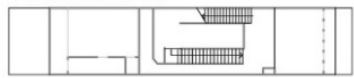

Third floor

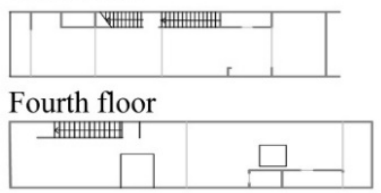

Figure 15: A basic contemporary tube house's plans and sections. 
Table 2: The visible relationship in the layout plans between the three basic types of tube houses.

\begin{tabular}{lllll}
\hline Type & Connectivity & Minimum & Average & Maximum \\
\hline Traditional tube house & First floor & 21 & 586 & 1412 \\
& Second floor & 20 & 438 & 917 \\
Semi-traditional tube house & First floor & 6 & 262 & 627 \\
& Second floor & 30 & 451 & 1014 \\
\multirow{5}{*}{ Contemporary tube house } & First floor & 31 & 378 & 690 \\
& Second floor & 65 & 1448 & 1962 \\
& Third floor & 48 & 899 & 1587 \\
& Fourth floor & 179 & 5969 & 9736 \\
\hline
\end{tabular}

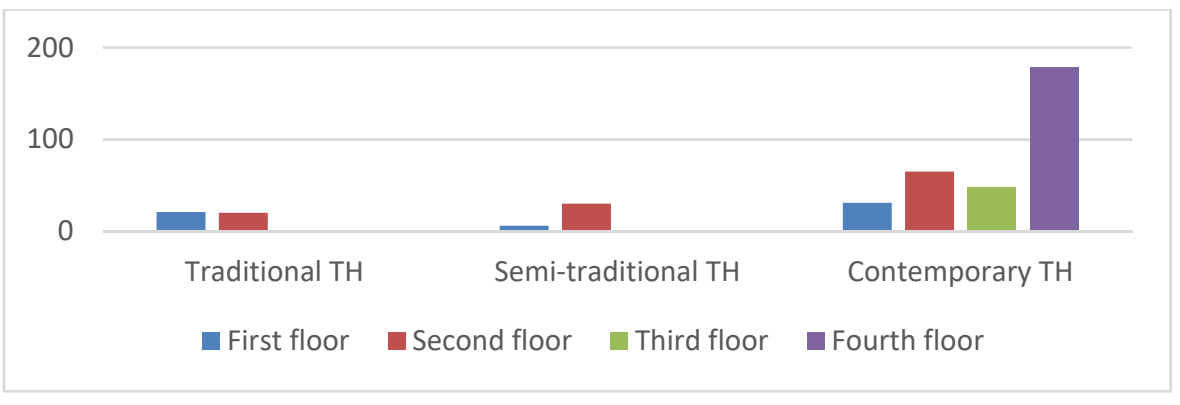

Figure 16: Minimum connectivity of above tube houses (TH).

\section{CONCLUSION}

1. This research has already investigated the current situation of tube house development in Hanoi, Vietnam.

2. The study has compared the traditional and contemporary tube house layout plans through different periods and come to the following conclusion: traditional tube houses have better natural lighting and ventilation, as well as a community connection and urban eco space.

3. The research has conducted a quantitative analysis of the visibility connection in tube house layout plans using Space Syntax.

4. The study has compared the visibility connections in tube house layout plans: among the three chosen houses, the visibility connections in modern tube houses are much higher than the traditional and semi-traditional tube houses.

\section{REFERENCES}

[1] Kinh Tế Việt Nam, Phạm Minh Chính, Vương Quân Hoàng, Nhà xuất bảnChính trị Quốc gia, p. 469.

[2] National Bureau of Statistics of Vietnam, Vietnam urbanization progress, 2013.

[3] Hanoi Statistics Bureau, Hanoi Statistical Yearbook 2013, pp. 65-89.

[4] Hanoi Planning Department, Hanoi Administrative Urbanization 2015, pp. 32-76.

[5] Hanoi Planning Department, Master Plan of Hanoi Project 2013, Thuyết minh bản đầy đủ, pp. 22-23. 
218 Sustainable Development and Planning IX

[6] Hanoi Urban Management Department, Statistic Book 2015, pp. 31-52.

[7] Hanoi chu ki cua nhung doi thay, Nhà xuất bản khoa hoc va ki thuat, pp. 257-261.

[8] Hanoi Old Quarter Management Department, Cai tao xay dung moi trong pho co Ha Noi.

[9] Jaepil Choi, Youngjun Choi, Youngwoo Kim, Junekyung Kang, Apartment Prototype with Enhanced Natural Ventilation System for Vietnam. 